\title{
Mitigation of persistent current effects in the RHIC superconducting magnets
}

\author{
C. Liu, ${ }^{1,{ }^{*}}$ D. Bruno $\odot,{ }^{1}$ A. Marusic $\odot,{ }^{1}$ M. Minty, ${ }^{1}$ P. Thieberger, ${ }^{1}$ and X. Wang $\odot^{2}$ \\ ${ }^{1}$ Brookhaven National Laboratory, Upton, New York 11973, USA \\ ${ }^{2}$ Lawrence Berkeley National Laboratory, Berkeley, California 94720, USA
}

(Received 8 August 2019; published 26 November 2019)

\begin{abstract}
Persistent currents in superconducting magnet introduce magnetic errors especially at low operating fields. In addition, their decay causes magnetic field variations and therefore drifts of the beam orbits, tunes, and chromaticities. To reduce field errors and suppress magnetic field variations, a new magnetic cycle was proposed for the low-energy beam operation at the Relativistic Heavy Ion Collider (RHIC). In the new magnetic cycle, the magnet current oscillates around the nominal operating current with diminishing amplitude a few times before it settles. The new magnetic cycle has been demonstrated experimentally to reduce field errors and the amplitude of magnetic field variations significantly and is essential for the ongoing RHIC Beam Energy Scan II (BES-II) program. This article will present beam-based experimental studies of the persistent current effects with the new magnetic cycle, and discuss its application in RHIC.
\end{abstract}

DOI: $10.1103 /$ PhysRevAccelBeams.22.111003

\section{INTRODUCTION}

Persistent current effects can lead to operational difficulties in accelerators based on superconducting magnets [1-4], especially at relatively low operating currents. Persistent currents produce magnetic field errors inside the aperture of superconducting magnets. The magnetic field errors can be described by the Fourier series expansion of the radial field component

$$
B_{y}+i B_{x}=B_{\text {ref }} \sum_{1}^{\infty}\left(b_{n}+i a_{n}\right)\left(\frac{x+i y}{r_{0}}\right)^{n}
$$

where $B_{x}$ and $B_{y}$ denote the horizontal and vertical components of the magnetic field respectively, $B_{\text {ref }}$ is the reference field, $x$ and $y$ are the horizontal and vertical position, $r_{0}$ is the reference radius, and $i$ is the imaginary unit. Here, $n=1$ refers to the dipole component. The reference radius chosen for most of the RHIC magnets is $25 \mathrm{~mm}$. The multipoles are usually normalized to the main field component and expressed in unit of $10^{-4}$. For instance, $b_{3}$ for a dipole magnet denotes the relative deviation of the sextupole field with respect to the main (dipole) field component, $b_{3}=B_{3} / B_{1} \times 10^{4}$. The magnetic field errors due to persistent currents [5] produce

*cliu1@bnl.gov

Published by the American Physical Society under the terms of the Creative Commons Attribution 4.0 International license. Further distribution of this work must maintain attribution to the author(s) and the published article's title, journal citation, and DOI. strong sextupole $\left(b_{3}\right)$ and decapole $\left(b_{5}\right)$ components in the main dipole magnets, octupole $\left(b_{4}\right)$, and 12-pole $\left(b_{6}\right)$ components in the quadrupole magnets of the RHIC accelerator system, which can limit the dynamic aperture of the accelerators. In the proton ring of the HadronElectron Ring Accelerator (HERA), a multipole field correction system [2] was implemented to improve the dynamic aperture by reducing the magnetic errors.

In addition, the decay of magnetic field errors, as a result of flux creep [6] and the interaction between the redistribution of currents in the superconducting cables comprising the magnets and the filament magnetization [7], cause drifts of the beam orbits, tunes and chromaticities. The time-dependence of magnetic field errors in superconducting accelerator magnets was first observed at the Tevatron in 1987 [1], and subsequently in HERA [8], RHIC [9], and LHC [10]. The effect on beam lifetime from the decay was observed to be most detrimental during beam injection because the relative contribution of persistent current is the strongest. To reduce the drifts of beam parameters, pre-programmed compensation [3] of the dipole, quadrupole, and sextupole fields was implemented.

The hysteresis of superconducting magnets can be well explained and modeled by the persistent currents in superconductor [11,12], which implies that contribution from iron hysteresis is negligible, even though the iron yoke in the RHIC superconducting dipole magnets contributes $\sim 30 \%$ of the total magnetic field [13] when it is below or near saturation. Therefore, the static change of field due to different magnetic cycles (see subsection III B) is attributed to persistent currents in this report. Regarding field variation in time (see subsection III C), it is certain that 
TABLE I. BES-II beam energies and corresponding dipole currents and field strength.

\begin{tabular}{lccccc}
\hline \hline E $(\mathrm{GeV} /$ nucleon) & 9.8 & 7.3 & 5.75 & 4.59 & 3.85 \\
Dipole current (A) & 472.7 & 351.5 & 275.7 & 218.1 & 180.8 \\
Dipole field (T) & 0.334 & 0.248 & 0.194 & 0.154 & 0.127 \\
\hline \hline
\end{tabular}

the permeability of the iron is constant over the experimental measurement period and therefore there is no contribution from iron yoke.

Persistent current effects are especially strong for the Beam Energy Scan II (BES-II) physics program at RHIC [14], where the gold beams collide at beam energy from 9.8 to as low as $3.85 \mathrm{GeV} /$ nucleon (Table I) with superconducting magnets operating outside of the nominal design range. To compensate the sextupole field errors induced by persistent currents, the polarities of some sextupole magnets had to be reversed during past operation at low energy [15]. In addition, significant time was spent for the magnetic field to reach a steady state for beam operation.

To combat persistent current effects, a new demagnetization magnet cycle [16] was proposed acting on all RHIC superconducting dipoles and quadrupoles. The magnet current oscillates around the operating current with diminishing amplitude a few times in the demagnetization cycle, similar to a "degaussing cycle" [17] which was exercised on one of the LHC magnets and then magnetic measurements were performed. The magnets after the degaussing cycle are in a magnetization state which is equally sensitive to small increments and decrements of the superimposed field. Beam-based studies at the RHIC injection energy in 2018 demonstrated that the new cycle reduced both the

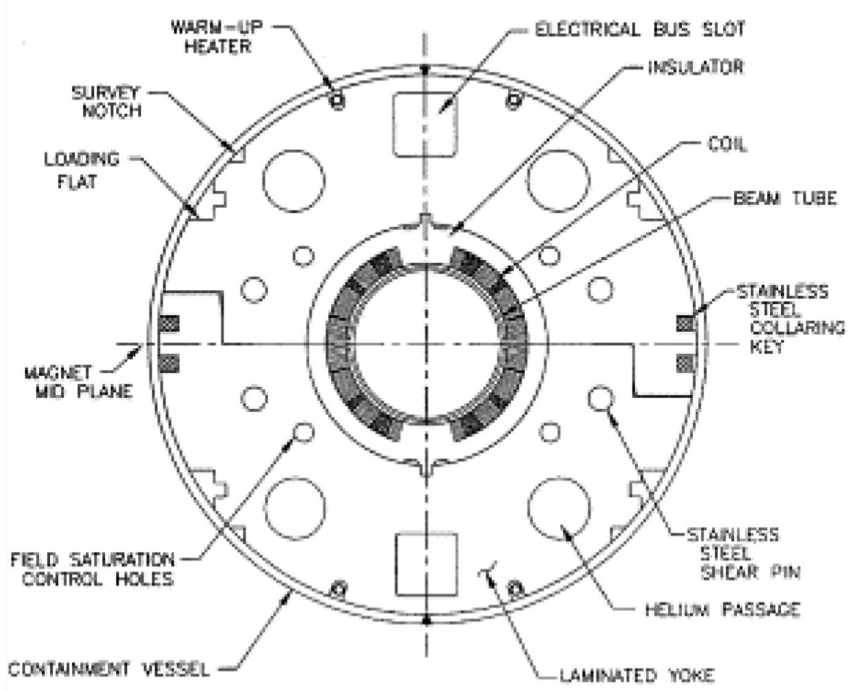

sextupole field error and its variation in time significantly. The proposal and beam studies of demagnetization magnet cycle motivated the magnetic simulation which was able to confirm part of the experimental observation, the change of the dipole field and the sextupole field errors.

This paper is structured as follows. The hysteresis cycle and decay of persistent currents in superconducting magnets are introduced in Sec. II. The calculated persistent current effects in a RHIC main dipole magnet are compared for the conventional and demagnetization cycles. The experimental test of the demagnetization magnetic cycle, beam-based studies of persistent current induced field errors and beam-based study of persistent current decay, are detailed in Sec. III. Section IV summarizes the experimental results and discusses application of the demagnetization cycle in RHIC and other accelerators.

\section{HYSTERESIS CYCLE AND ITS IMPACT ON THE PERSISTENT CURRENT EFFECTS IN RHIC SUPERCONDUCTING DIPOLE MAGNETS: CALCULATION BASED ON THE CRITICAL-STATE MODEL}

Persistent currents cause hysteresis of magnetic fields in the aperture of superconducting accelerator magnets $[18,19]$. According to the critical-state model [20,21], when the applied field changes, persistent currents will be induced inside the superconducting filament opposing the field change $[18,19,22]$. Although the NbTi filament will be fully penetrated by applied field at the scale of $0.1 \mathrm{~T}$ for the strands used in RHIC arc dipole and quadrupole magnets (Fig. 1) [23], complex profiles of the persistent currents can form inside the filament depending on the magnitude and history of the applied field. Therefore, the

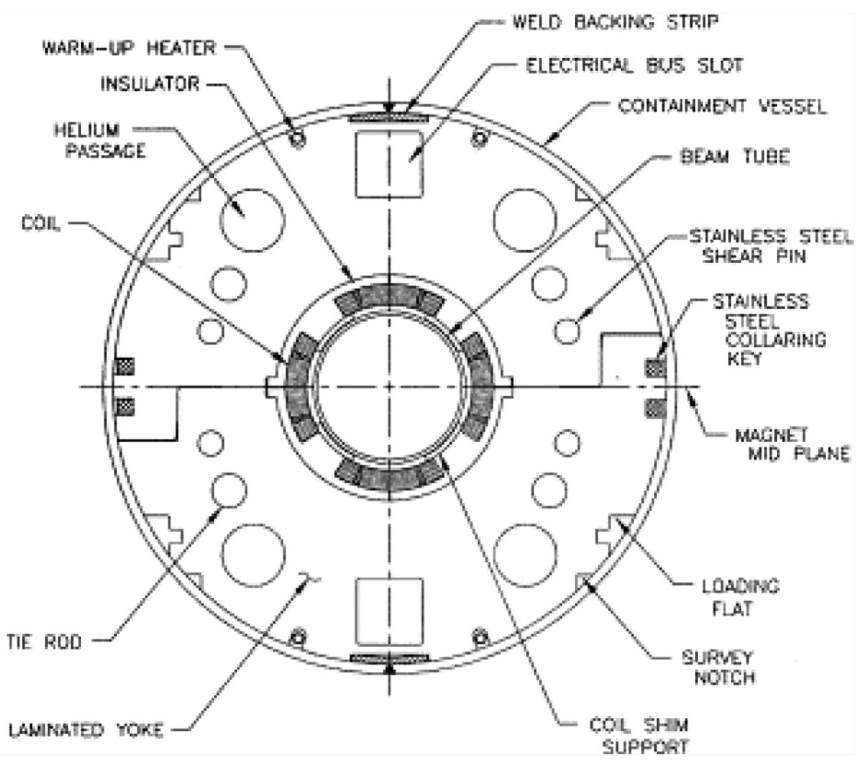

FIG. 1. The left plot shows the cross-section of the main dipole magnet. The right plot shows the cross section of the main quadrupole magnet. 


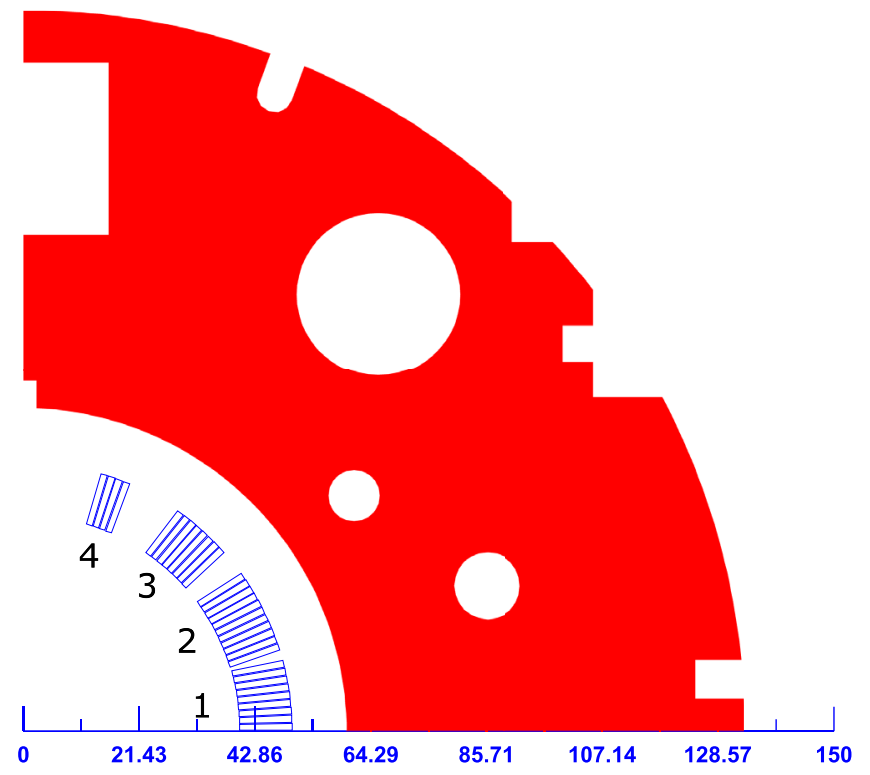

FIG. 2. The cross section of the RHIC main arc dipole magnet. The first quadrant with four coil blocks is shown (Unit: mm).

strand magnetization and the resulting field errors can be tuned by varying the powering scheme/cycle for accelerator magnets.

The magnetic field of superconducting magnets decay because of persistent currents and coupling currents (currents across superconducting filaments/strands) decay once the external main field stops ramping [7]. Flux creep induces a decrease of the critical current density and produces a logarithmic decay. Interfilament and interstrand coupling currents develop when the external field changes, and decay when the external field stays constant. Boundary induced coupling currents result in different currents in strands, the redistribution of which can affect the magnetization due to changes of the local field $[12,24]$.

To provide guidance on what to expect when switching from the conventional to the demagnetization cycle, we

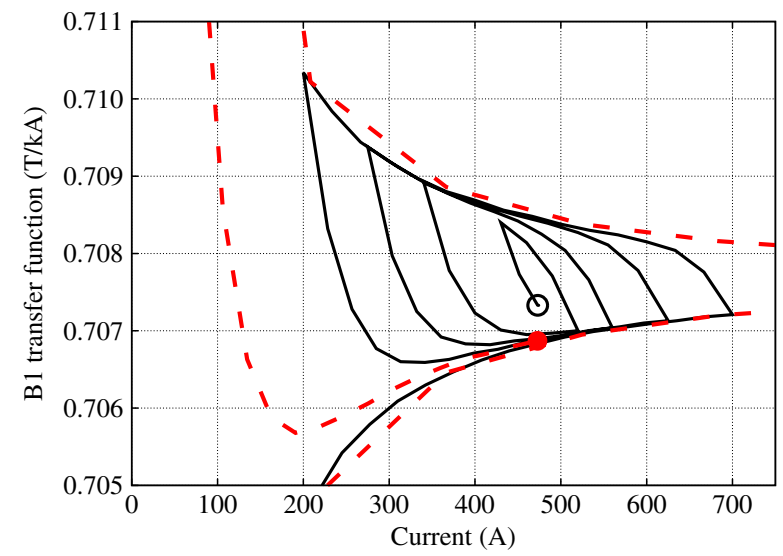

TABLE II. The calculated dipole transfer function and $b_{3}$ at the end of the conventional and the demagnetization cycles. The relative change with respect to the conventional cycle is given for the dipole transfer function.

\begin{tabular}{lcccc}
\hline \hline & Unit & Conventional & New & Change \\
\hline$B_{1}$ transfer function & $\mathrm{T} \mathrm{kA}^{-1}$ & 0.7069 & 0.7073 & $+0.065 \%$ \\
$b_{3}$ at $R_{\text {ref }}=25 \mathrm{~mm}$ & Unit & -17.6 & -12.8 & +4.8 \\
\hline \hline
\end{tabular}

calculated the persistent current effects in RHIC superconducting dipole magnets using ROXIE $[25,26]$ based on the critical-state model $[20,21]$. We considered the field errors in the straight part of the magnet based on the asdesigned magnet cross section (Fig. 2). Uniform critical current density $J_{\mathrm{c}}(B)$, based on Bottura's formula [27], was applied to all the NbTi strands. Therefore, the persistent current induced multipoles appear only in the allowed terms such as $B_{1}$ and $b_{3}$ for the dipole magnet. The calculation considered the nonlinear $B-H$ curve for the iron yoke but not the hysteresis effect [28].

Figure 3 shows the calculated transfer function of the dipole field and $b_{3}$ as a function of dipole magnet current for the conventional (Fig. 6) and the demagnetization cycles (Fig. 5). The values of the dipole transfer function and $b_{3}$ at the end of each cycle are given in Table II.

To illustrate the impact of the new cycle on the persistent current effects, we compare the profiles of the persistent current in a superconducting NbTi filament at the end of two cycles. We choose the filament in the NbTi strand at the top left corner of the coil block No. 2 (Fig. 2) because it is one of the strands that contribute most to the persistent current induced $b_{3}$.

We discretize the $6 \mu \mathrm{m}$ diameter NbTi filament [23] into uniform grids. The current density distribution is then computed self-consistently as a function of applied magnetic induction based on the magnet transport current and the self-field from each grid. To solve the critical-state

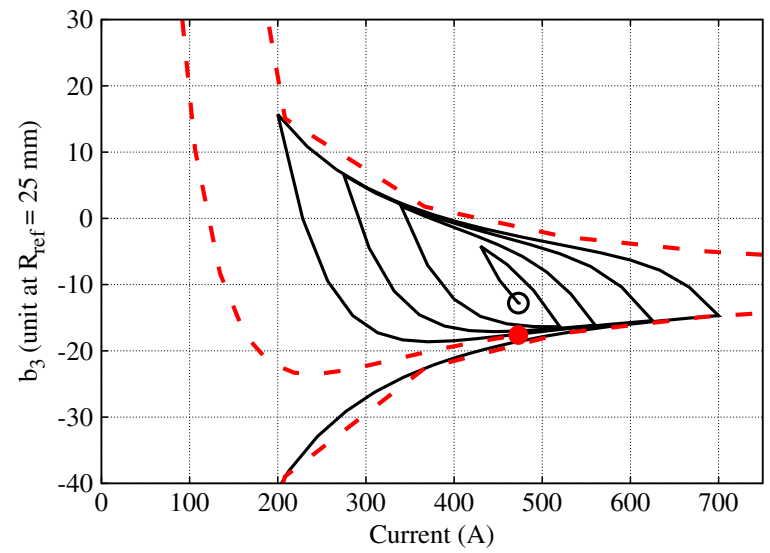

FIG. 3. The calculated dipole transfer function and normal sextupole component $\left(b_{3}\right)$ from the conventional cycle (red dashed line) and the demagnetization cycle (black solid line). The points denote the resulting values at the end of each cycle. $R_{\text {ref }}=25 \mathrm{~mm}$. 

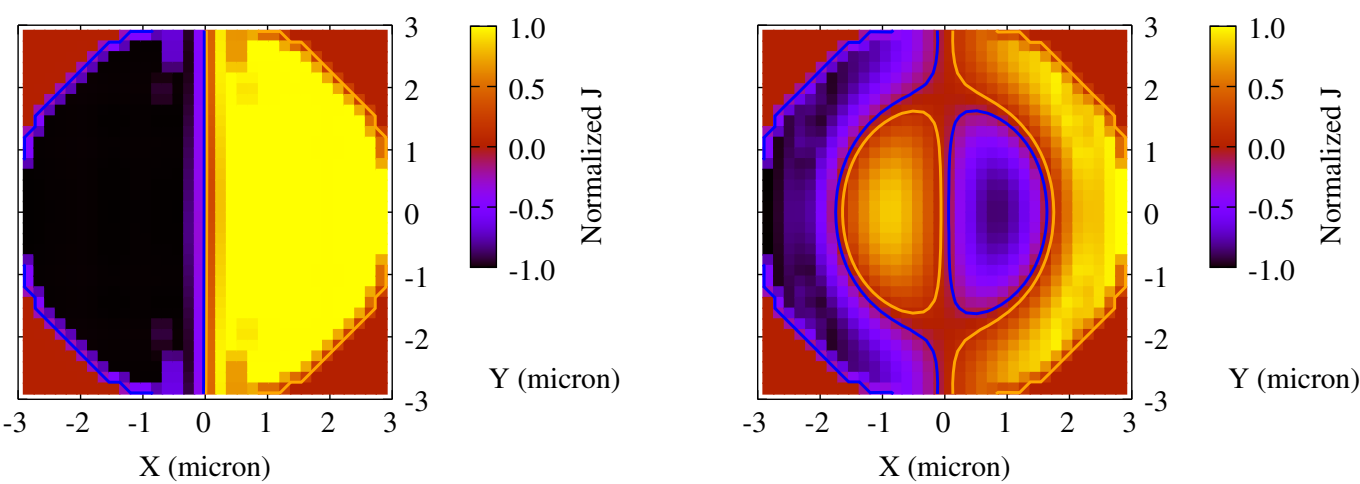

FIG. 4. Distribution of the current density in a NbTi filament at the end of two cycles. Left: the conventional cycle. Right: the demagnetization cycle. The current density is normalized to the $J_{\mathrm{c}}$ of the NbTi conductor at $5 \mathrm{~T}, 4.2 \mathrm{~K}$ [27]. Contour lines are for +0.1 (orange) and -0.1 (blue) of the normalized current density.

problem, we used Prigozhin's approach [29] with the quadratic programming solver from the GALAHAD package [30,31]. Transport current in the filament is not considered as it is negligible compared to the critical current.

The conventional and demagnetization cycles clearly lead to different current density distribution in the superconducting filament (Fig. 4), consistent with the earlier study for the LHC dipole magnet [17]. Based on the current density distribution as shown in Fig. 4, we calculated and found that the magnetization of the NbTi strand $(-3.7 \mathrm{mT})$ at the end of the demagnetization cycle decreased by $57 \%$ from the conventional cycle $(-8.6 \mathrm{mT})$.

\section{EXPERIMENTAL STUDIES ON PERSISTENT CURRENTS WITH A DEMAGNETIZATION CYCLE AT INJECTION ENERGY}

\section{A. Experiment overview}

A new demagnetization cycle for RHIC dipoles and quadrupoles, with the currents oscillating a few times with a diminishing amplitude before settling at the operating current, is shown in Fig. 5. For comparison, the conventional magnet cycle, with the current increased to high amplitude then decreased to a low value then returning to the operating current, is shown in Fig. 6. All experiments reported here were performed at the nominal RHIC gold beam injection energy of $9.8 \mathrm{GeV} /$ nucleon (Table I).

The RHIC dipoles and quadrupoles magnets were ramped through the demagnetization cycle twice. After the first cycle, the dipole, quadrupole and the sextupole current were adjusted to compensate the difference in magnetic fields introduced by the demagnetization cycle. After the second cycle, the variation of magnetic fields were measured by monitoring the drift of beam orbit, tunes, and chromaticities. For operation, only one demagnetization cycle is required to reproduce stable machine conditions which do not vary with more demagnetization cycles.

\section{B. Change of magnetic fields induced by the demagnetization cycle}

In this subsection, we present and analyze first the change of high-order magnetic field errors and then the change of low-order magnetic field components. The highorder magnetic field errors of concern are the sextupole $\left(b_{3}\right)$ and decapole $\left(b_{5}\right)$ components from RHIC superconducting dipoles, and the octupole $\left(b_{4}\right)$ component from superconducting quadrupoles. These components are monitored by comparing measurements of first, second, and third order chromaticities respectively. The low-order magnetic field errors alter the dipole and quadrupole fields. These changes were inferred from the measured beam average orbit in the dispersive arc sections and from errors in the $\beta$-functions ( $\beta$-beat), respectively. The observations/ corrections described in this subsection were made after

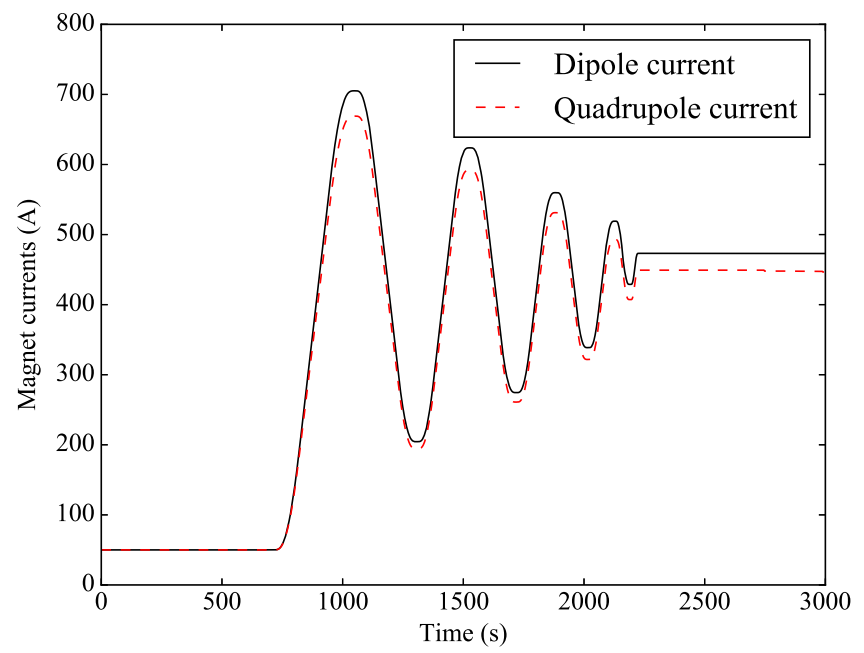

FIG. 5. Demagnetization magnetic cycle: RHIC superconducting dipole and quadrupole magnet currents are ramped up and down several times, with intermediate dipole current points at [50, 700, 200, 625, 275, 560, 340, 520, $430 \mathrm{~A}]$, before being held constant at the operating currents for beam injection. 


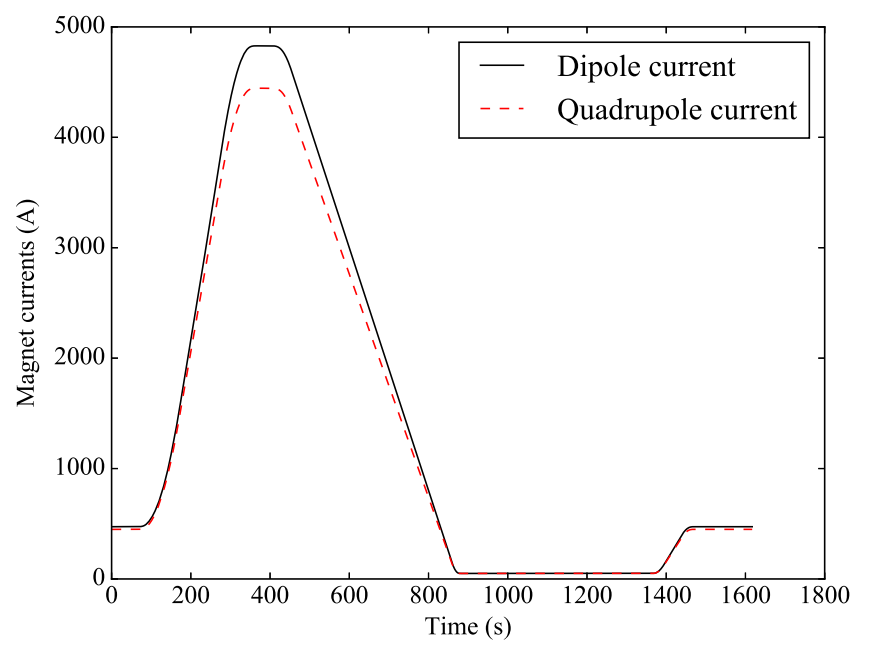

FIG. 6. Conventional magnetic cycle: RHIC superconducting dipole and quadrupole magnet currents are ramped up to currents for top energy, back down to $50 \mathrm{~A}$, then up to the operating values for beam injection.

the fields reached equilibrium, namely when persistent currents decay was negligible.

\section{Reduction of sextupole field errors in the dipole magnets}

The change of sextupole components in the superconducting dipoles introduced by the demagnetization cycle will be deduced first. At injection energy $(9.8 \mathrm{GeV} /$ nucleon) which is below transition energy $(\sim 24 \mathrm{GeV} /$ nucleon), chromaticities [the ratio between betatron tune shift and relative momentum deviation [5], $\delta \nu /(\delta p / p)]$ are set to be $\sim-5$ for beam stability using sextupole magnets. The contributions to chromaticities consist of the natural chromaticity from quadrupoles $\left(Q_{\text {natural }}\right)$, sextupole components in dipoles $\left(b_{3}\right)$ and compensation from sextupole magnets $\left(Q_{c}\right)$

$$
k * b_{3}+Q_{\text {natural }}+Q_{c}=-5,
$$

where $b_{3}$ is the average sextupole component in RHIC superconducting dipoles, $k=4.6$ is the scale factor [32] for converting sextupole component to beam chromaticity unit, $Q_{\text {natural }}$ is the natural chromaticity from the quadrupoles and $Q_{c}$ is the compensation from sextupole magnets.

After the demagnetization cycle, to keep the measured chromaticity at $\sim-5$ the sextupole compensation was lowered by 20 units in the horizontal plane and increased about by the same amount in the vertical one. As will be shown in subsection III B 3, the change of the quadrupole field was not significant, therefore we may assume that the natural chromaticities and the scale factor were little changed after the demagnetization cycle and write

$$
k * b_{3, n}+Q_{\text {natural }}+\left(Q_{c}-20\right)=-5,
$$

where $b_{3, n}$ is the new average sextupole component in RHIC superconducting dipoles after the demagnetization cycle. Combining Eqs. (2) and (3), the new sextupole component after the demagnetization cycle $\left(b_{3, n}\right)$ is -1.04 . Therefore, the sextupole component from the RHIC superconducting dipoles was reduced from -5.39 with the conventional magnetic cycle to -1.04 with the demagnetization cycle. The observed change of $b_{3}$ from the beam measurements was consistent with the expected +4.8 units from the calculation (Table II). The $b_{3}$ values shown in Table II were calculated based on the as-designed RHIC main dipole magnet. The actual as-built magnets have different geometric errors. The difference in the calculated and measured $b_{3}$ values was due to the geometric errors in RHIC dipole magnets.

\section{Reduction of the second and third order chromaticities}

The demagnetization cycle was proven to reduce the high-order errors significantly based on the comparison of the absolute value of the second $\left(Q^{\prime \prime}\right)$ and third order chromaticities $\left(Q^{\prime \prime \prime}\right)$. Measured $Q^{\prime \prime}$ and $Q^{\prime \prime \prime}$ with statistic errors of multiple measurements are compared in Table III for the demagnetization and conventional magnetic cycles.

The octupole and decapole correctors were not used at injection, neither for the conventional nor for the demagnetization cycle. Therefore, the measurement of $Q^{\prime \prime}$ and $Q^{\prime \prime \prime}$ are the direct representation of the high-order field errors in the dipole and quadrupole magnets.

\section{Change of dipole and quadrupole fields}

The changes of dipole and quadrupole magnetic fields introduce changes of the beam orbits, betatron tunes and Twiss parameters. These changes were compensated by adjusting the dipole and quadrupole magnet currents. Here the magnitude of the changes of beam average orbit and $\beta$-beat are presented.

The horizontal orbit after the demagnetization cycle shifted inwards by $1.8 \mathrm{~mm}$ (Fig. 7) in the dispersive

TABLE III. Comparison of the second and third order chromaticities measured with the conventional and the demagnetization cycles.

\begin{tabular}{lrrrr}
\hline \hline Cycle & $Q_{h}^{\prime \prime}$ & \multicolumn{1}{c}{$Q_{v}^{\prime \prime}$} & \multicolumn{1}{c}{$Q_{h}^{\prime \prime \prime}$} & \multicolumn{1}{c}{$Q_{v}^{\prime \prime \prime}$} \\
\hline Conventional & $459 \pm 806$ & $-478 \pm 815$ & $4.7 \mathrm{E} 6 \pm 6.7 \mathrm{E} 6$ & $-1.5 \mathrm{E} 6 \pm 3.5 \mathrm{E} 6$ \\
Demagnetization & $58 \pm 345$ & $31 \pm 188$ & $-1.9 \mathrm{E} 6 \pm 3.6 \mathrm{E} 6$ & $-1.5 \mathrm{E} 5 \pm 3.0 \mathrm{E} 5$ \\
\hline \hline
\end{tabular}




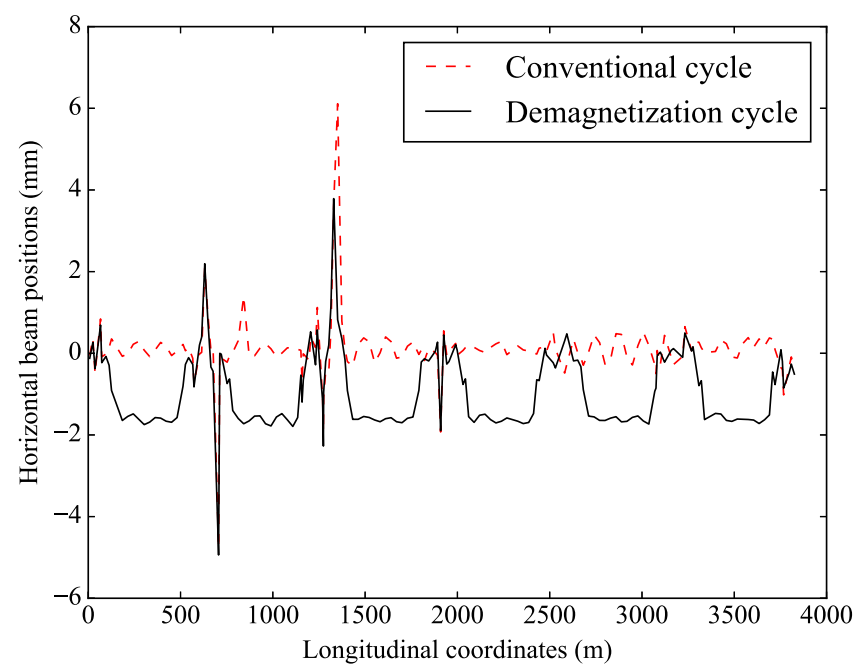

FIG. 7. The horizontal orbits shifted inwards by $1.8 \mathrm{~mm}$ in the dispersive regions due to the change of RHIC dipole magnet field with the demagnetization cycle.

regions with respect to the otherwise centered orbit after a conventional magnetic cycle. The orbit shift may be explained by the change of RHIC dipole field after implementation of the demagnetization cycle. A relative adjustment of RHIC dipole field of $\delta B / B=-0.0448 \%$ was required to recenter the beam orbit. This observation was consistent with the expected $0.065 \%$ increase in the dipole field from the demagnetization cycle (Table II).

The magnitude of the $\beta$-beat was $\sim 15 \%$ peak-to-peak, comparable to the ones measured with the conventional magnetic cycle and therefore no compensation was applied. This observation seems indicating that persistent current effects have negligible impact on the main field of the RHIC quadrupole magnets. Further investigation of this will be continued in the future.

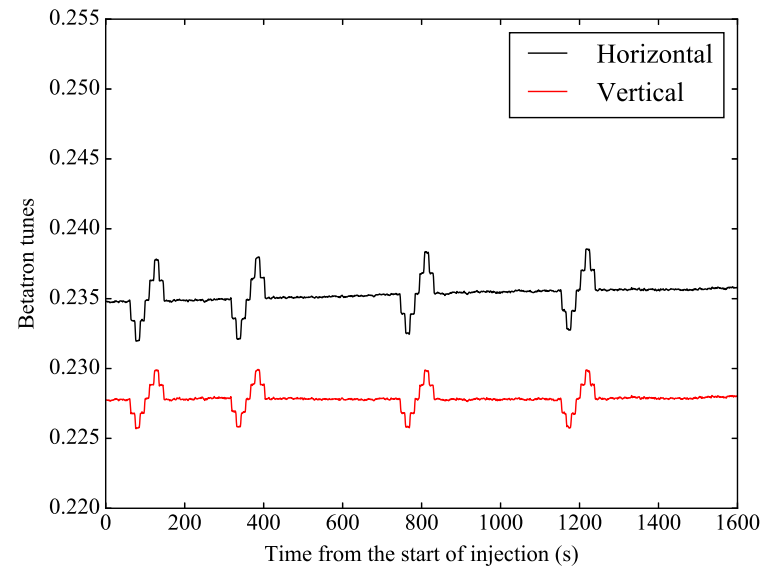

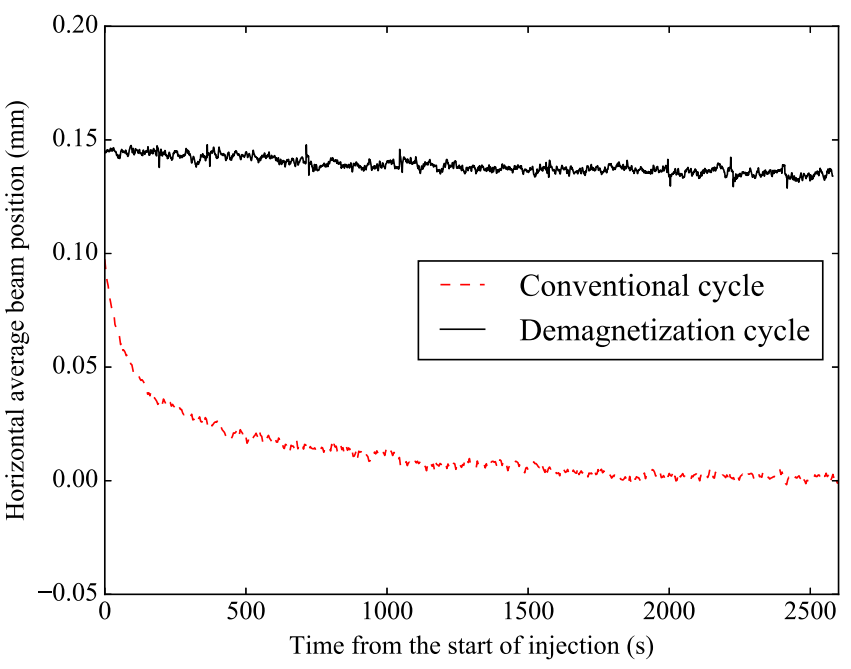

FIG. 8. Comparison of the horizontal average beam position drifts, due to persistent currents decay in superconducting dipoles, with the conventional cycle (the blue curve) and the demagnetization cycle (the green curve).

\section{Beam-based measurement of drifts of the magnetic fields}

Drifts of the beam orbits, betatron tunes and chromaticities introduced by the decay of persistent and coupling currents result in a reduced beam lifetime. In the following, the beam-based measurements of the drifts of these parameters with the conventional and the demagnetization cycles are presented.

The drifts of the dipole main field cause a drift of the average beam position in the dispersive arc sections. The magnitude of the drifts are compared in Fig. 8 for the conventional and demagnetization cycles. The drift of the average beam position was reduced by a factor of $\sim 10$ with the demagnetization cycle.

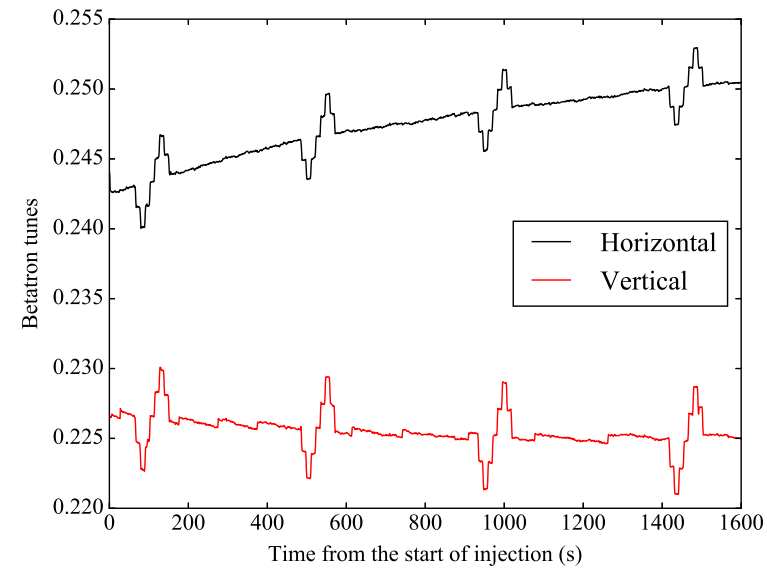

FIG. 9. The left plot shows the drift of the horizontal and vertical betatron tunes after the demagnetization cycle. The right plot shows the drift of the horizontal and vertical betatron tunes after the conventional cycle. The sawtoothlike excursions of the betatron tunes resulted from the rf frequency changes used to measure chromaticities. 

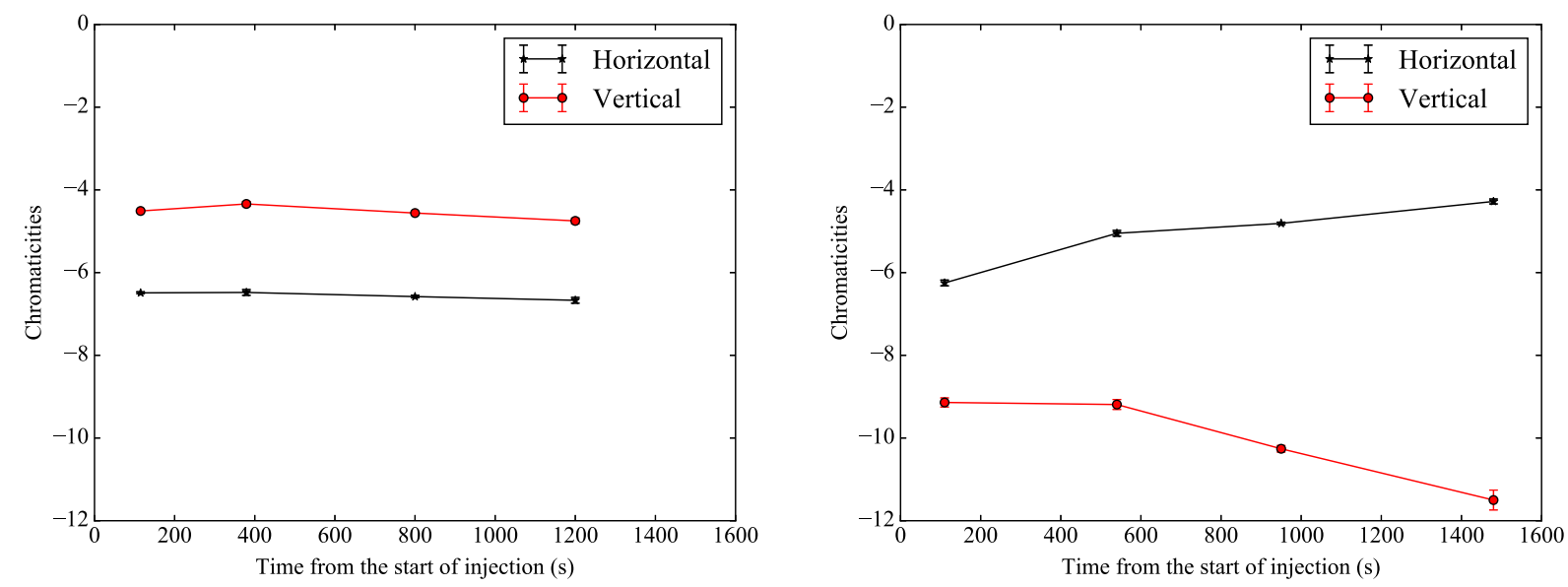

FIG. 10. The left plot shows the measured beam chromaticities after the demagnetization cycle. The right plot shows the drift of measured beam chromaticities after a conventional cycle.

The drifts of the quadrupole main field causes a drift of the betatron tunes. The magnitude of the drifts are compared in Fig. 9 for the demagnetization and conventional cycles. Unlike the conventional cycle, the betatron tunes after the demagnetization cycle did not show variations. The sawtooth-like excursions of the betatron tunes is a result of the rf frequency changes used to measure chromaticities.

The drift of the sextupole field causes a drift of beam chromaticities. The measured beam chromaticities over a $\sim 20$ minutes period with the demagnetization cycle and with the conventional cycle are shown in the left and right plot of Fig. 10 respectively. The drifts of beam chromaticities with the conventional cycle were studied more systematically at RHIC [9] in 2001. The few new measurements (Fig. 10 right) showed a logarithmic variation

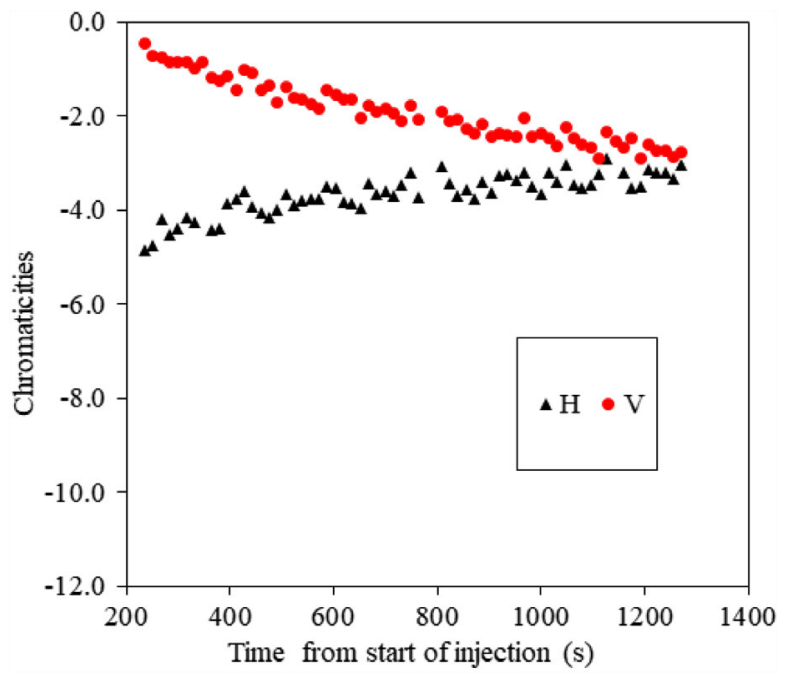

FIG. 11. Drift of the measured beam chromaticities, reproduced from Fig. 3 in Ref. [9], after a conventional cycle. with time the same as the old ones, reproduced in Fig. 11. The beam chromaticities stayed almost constant with the demagnetization cycle.

\section{SUMMARY}

A demagnetization magnet cycle, with magnet current oscillating a few times with diminishing amplitude around the operating current before settling, was proposed at RHIC to combat the persistent current effects in superconducting magnets. Beam-based studies demonstrated substantial reduction of persistent current induced magnetic field errors and their decay. The sextupole component in dipoles from persistent current was significantly reduced. The second and third order chromaticities were measured to be smaller with the demagnetization cycle than those with the conventional cycle. The change of dipole field, which was measured by orbit shift, was compensated by adjusting the main field. The change of quadrupole field, which was measured by $\beta$-beat, were observed to be nonsubstantial. The drift of the dipole field, which was measured by drift of the average beam position, was reduced by a factor of $\sim 10$ with the demagnetization cycle. The drift of quadrupole field, which was measured by the drift of betatron tunes, was reduced by a factor of $\sim 8$ with the demagnetization cycle. The sextupole components, inferred by the measured chromaticities, were mostly constant compared to a logarithmic decay with the conventional cycle.

The observed benefits of the demagnetization cycle include improvement of beam lifetime due to reduction of field errors, and more stable accelerator conditions due to suppressed persistent current decay. These benefits are expected to also improve performance of RHIC during continuation of the Beam Energy Scan II program [33], for which the magnets must be operated at currents much lower than at the nominal RHIC injection energy. 
In this report, the demagnetization cycle to remove persistent current effects were studied at fixed nominal magnetic field. To investigate the variation of persistent currents when the magnetic field starts ramping up (so called snapback effect [34]), a future study of persistent current with the demagnetization cycle followed by acceleration has been planned in RHIC. If successful, the demagnetization cycle can potentially be applied to existing and future superconducting accelerators designed for a wide energy range.

\section{ACKNOWLEDGMENTS}

This research was supported by Brookhaven Science Associates, LLC under Contract No. DE-SC0012704 with the U.S. Department of Energy. The work at Lawrence Berkeley National Laboratory was supported by the Director, Office of Science, and Office of High Energy Physics of the U.S. Department of Energy under Contract No. DE-AC02-05CH11231.

[1] D. A. Finley et al., Time dependent chromaticity changes in the Tevatron, in Proceedings of the 1987 Particle Accelerator Conference (IEEE, Washington, DC, 1987), pp. 151-153.

[2] F. Willeke and F. Zimmermann, The impact of persistent current field errors on the stability of the proton beam in the HERA proton ring, in Proceedings of the 1991 Particle Accelerator Conference, San Francisco, CA, 1991 (IEEE, New York, 1991), pp. 2483-2485.

[3] D. A. Herrup, W. Kinney, M. J. Lamm, and A. Mokhtarani, Compensation of time-dependent persistent current effects in superconducting synchrotrons, Phys. Rev. E 49: 5660 (1994).

[4] B. J. Holzer, Impact of persistent currents on accelerator performance, Part. Accel. 55, 215 (1996).

[5] A. W. Chao et al., Handbook of Accelerator Physics and Engineering (World Scientific, Singapore, 2013).

[6] P. W. Anderson, Theory of Flux Creep in Hard Superconductors, Phys. Rev. Lett. 9, 309 (1962).

[7] S. Amet et al., Persistent and coupling current effects in the LHC superconducting dipoles, IEEE Trans. Appl. Supercond. 13, 1239 (2003)

[8] H. Brück et al., Time dependence of persistent current effects in the superconducting HERA magnets, in 11th International Conference on Magnet Technology (MT-11) (Springer, New York, 1990), pp. 141-146.

[9] W. Fischer, A. Jain, and S. Tepikian, Beam-based measurements of persistent current decay in the Relativistic Heavy Ion Collider, Phys. Rev. Accel. Beams 4, 041002 (2001).

[10] N. Aquilina, M. Lamont, M. Strzeclzyk, E. Todesco, and N. Sammut, Chromaticity decay due to superconducting dipoles on the injection plateau of the Large Hadron Collider, Phys. Rev. Accel. Beams 15, 032401 (2012).

[11] X. Wang et al., Persistent current effects in RHIC arc dipole magnets operated at low currents. Proc. of
NAPAC2016, Chicago, IL, USA, pp. 170-173, http:// accelconf.web.cern.ch/AccelConf/napac2016/papers/ mopob49.pdf.

[12] C. Völlinger, Superconductor magnetization modeling for the numerical calculation of field errors in accelerator magnets, Ph.D. thesis, Berlin, Tech. U., 2002.

[13] R. Gupta, USPAS Course on Superconducting Accelerator Magnet (US Particle Accelerator School, University of California Santa Barbara, 2003), https://www.bnl.gov/ magnets/staff/gupta/scmag-course/uspas03/RG03/.

[14] M. M. Aggarwal et al., An experimental exploration of the QCD phase diagram: The search for the critical point and the onset of de-confinement, arXiv:1007.2613.

[15] C. Montag et al., Experience with low-energy gold-gold operations in RHIC during FY 2010, Technical Report No. BNL C-A/AP/435, 2011.

[16] P. Thieberger, Can we demagnetize ("degauss") the effect of persistent currents in RHIC?, RHIC APEX workshop, 2013 (unpublished).

[17] W. V. Delsolaro, L. Bottura, M. Haverkamp, A. Kuijper, L. Bottura, M. Haverkamp, and A. Kuijper, Degaussing and decay reduction in the short superconducting dipole models for the LHC, IEEE Trans. Appl. Supercond. 12, 291 (2002).

[18] K. H. Mess, P. Schmüser, and S. Wolff, Superconducting Accelerator Magnets (World Scientific, Singapore, 1996), ch. 6 .

[19] M. N. Wilson, Superconducting Magnets (Clarendon Press, Oxford, 1983).

[20] C. P. Bean, Magnetization of Hard Superconductors, Phys. Rev. Lett. 8, 250 (1962).

[21] Y. B. Kim, C. F. Hempstead, and A. R. Strnad, Critical Persistent Currents in Hard Superconductors, Phys. Rev. Lett. 9, 306 (1962).

[22] A. Jain, Dynamic Effects in Superconducting Magnets (USPAS school, Phoenix, 2006).

[23] M. Anerella et al., The RHIC magnet system, Nucl. Instrum. Methods Phys. Res., Sect. A 499, 280 (2003).

[24] A.P. Verweij, Electrodynamics of superconducting cables in accelerator magnets, Ph.D. thesis, Twente U., 1997.

[25] C. Völlinger, M. Aleksa, and S. Russenschuck, Calculation of persistent currents in superconducting magnets, Phys. Rev. ST Accel. Beams Beams, 3, 122402 (2000).

[26] S. Russenschuck, Field Computation for Accelerator Magnets: Analytical and Numerical Methods for Electromagnetic Design and Optimization (John Wiley \& Sons, New York, 2010).

[27] L. Bottura, A practical fit for the critical surface of NbTi, IEEE Trans. Appl. Supercond. 10, 1054 (2000).

[28] D. Schoerling, Case study of a magnetic system for lowenergy machines, Phys. Rev. Accel. Beams 19, 082401 (2016).

[29] L. Prigozhin, The Bean model in superconductivity: Variational formulation and numerical solution, J. Comput. Phys. 129, 190 (1996).

[30] N. I. M. Gould, D. Orban, and P. L. Toint, GALAHAD, a library of thread-safe Fortran 90 packages for large-scale nonlinear optimization, ACM Trans. Math. Softw. 29, 353 (2003). 
[31] HSL, a collection of Fortran codes for large scale scientific computation, 2013. http://www.hsl.rl.ac.uk.

[32] Y. Luo et al., Modeling RHIC linear chromaticity with sextupole components in the arc main dipoles, Technical Report No. C-A/AP/276, 2007.

[33] C. Liu et al., Improving the luminosity for Beam Energy Scan II at RHIC, in Proc. IPAC'19, Melbourne, Australia,
May 2019, pp. 540-543, https://doi.org/10.18429/JACoWIPAC2019-MOPMP044.

[34] M. Haverkamp, Decay and snapback in superconducting accelerator magnets, Ph.D. thesis, Twente University Press (TUP), 2003. 